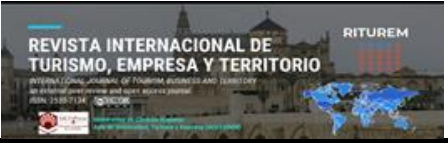

Cita bibliográfica: Mora Forero, J.A.; Vásquez Benítez, .L. y Gallego Vásquez, J.E. (2020). La formación universitaria en turismo: una perspectiva académica y laboral en Bogotá (Colombia). Revista Internacional de Turismo, Empresa y Territorio, 4 (2), 81-102. https://doi.org/10.21071/riturem.v4i2.12899

\title{
La formación universitaria en turismo: una perspectiva académica y laboral en Bogotá (Colombia).
}

\section{University training in tourism: an academic and labor perspective in Bogotá (Colombia)}

\author{
Jorge Alexander Mora Forero ${ }^{1}$ \\ Lylliana Vásquez Benítez ${ }^{2}$ \\ Jorge Enrique Gallego Vásquez ${ }^{3}$
}

\begin{abstract}
Resumen
Este artículo describe las relaciones y vínculos funcionales entre la formación profesional en hotelería y turismo en Bogotá (Colombia) y las necesidades de talento humano de las empresas del sector turístico y la inserción laboral del alumnado. La metodología de esta investigación ha sido de carácter cualitativo y los instrumentos de investigación se basaron en entrevistas estructuradas y semiestructuradas, proporcionadas por categorías de análisis previamente establecidas. Por un lado, se entrevistaron a los directores de las instituciones universitarias, para conocer el perfil profesional del egresado. Del mismo modo, se realizaron entrevistas a los directores de talento humano de los prestadores de servicios turísticos, con el fin de identificar las principales necesidades laborales de los cargos profesionales. Este trabajo de investigación se realizó en la ciudad de Bogotá D.C., durante el año 2018 y 2019 y se evidenciaron finalmente algunas discordancias entre la formación profesional académica y las necesidades de talento humano, entre las cuales se destaca la falta de práctica y el déficit en bilingüismo.
\end{abstract}

Palabras clave: Educación, turismo, competencias, talento humano

\footnotetext{
${ }^{1}$ Líder de investigación del programa Administración de Empresas Turísticas y Hoteleras, Corporación Universitaria Minuto de Dios - UNIMINUTO, Bogotá, Colombia. jorge.moraf@uniminuto.edu. https://orcid.org/0000-0001-7764-0370 * Autor para la correspondencia.

${ }^{2}$ Docente Investigadora Facultad de Educación, Corporación Universitaria Minuto de Dios - UNIMINUTO, Bogotá, Colombia. lvasqu16@uniminuto.edu. https://orcid.org/0000-0001-7867-2374.

${ }^{3}$ Docente Investigador Líder de línea de investigación en Gestión educativa y curricular para la formación integral de la Maestría en Educación de la Corporación Universitaria Minuto de Dios - UNIMINUTO, Bogotá, Colombia. jorge.gallego@uniminuto.edu. https://orcid.org/0000-0002-8674-2755
} 


\begin{abstract}
This article aims to describe the relationship in training of professionals in hospitality and tourism, with the human talent needs of companies in the tourism sector in Bogotá (Colombia). To this end, a bibliographic review was made about the researches that have studied the curricular plans in this discipline and their relationship with employment. The methodology of this research is qualitative; Structured and semi-structured interviews were conducted as research instruments. These were made to the directors of the university institutions, in order to know the Just graduated professional profile. A similar work was also carried out with the directors of human talent in the tourism services providers, to identify the main needs of the professional positions. This fieldwork was carried out in the city of Bogotá D.C., where some discrepancies between the academic professional training, as well as the needs of human talent are shown; among which was found, the lack of practice and the deficit in bilingualism.
\end{abstract}

Keywords: Education, tourism, skills, human resource

\title{
1. Introducción
}

El turismo es una actividad multidisciplinar y puede estudiarse, en consecuencia, desde distintos enfoques, entre los que se destacan los de las ciencias humanas y sociales (Fayos, 1997). El turismo, por otro lado, se ha ido imponiendo y consolidando como objeto de estudio y como disciplina académica en nuemerosas Instituciones Universitarias que ofrecen programas en distintos niveles de formación. Existe por lo tanto una oferta que busca cumplir con los requerimientos y necesidades del sector productivo a través de perfiles formativos, pero se evidencian frecuentemente distanciamientos entre dichas necesidades y la formación de los profesionales. Los empleados del sector turístico requieren de características específicas para su desempeño, pero para muchos autores como para Bañuls y Casado (2011) existe un bajo nivel educativo en los empleados en el sector debido a que abundan puestos de trabajo con muy escasos requerimientos formativos, lo que genera algunas brechas entre los procesos y contenidos de aprendizaje y las competencias demandadas por las empresas turísticas.

En materia educativa el turismo es un área de estudios compleja, debido a su naturaleza multidisciplinar (Korstanje, 2011; Nechar, 2007). Además, no son unánimes las construcciones curriculares en esta profesión, y las particularidades de cada uno de los programas presentan grandes diferencias. No obstante, se destaca el componente administrativo en las denominaciones de los programas académicos y los planteamientos curriculares que se establecen en los planes de estudio de los programas de turismo suele presentar frecuentemente un énfasis en el componente teórico y en algunos casos se trivializa el componente pragmático de la disciplina turística.

Por ello, es importante orientar las ofertas académicas hacia los nuevos retos que plantea la sociedad y el mundo de la empresa (Martínez, Ruiz y Valladares, 2012). En este caso, la academia es la llamada a realizar evaluaciones y cuestionamientos que permitan un estado de actualización y contextualización de las necesidades formativas en materia turística. En esta dinámica, las habilidades y competencias profesionales deben tener una 
relación directa con la práctica en empresas turísticas (Herrera, 2018). Rodrigues (2006) plantea que el turismo como disciplina profesional aún sigue siendo un área incipiente, de manera que para alcanzar un nivel de madurez debe existir una cooperación interinstitucional con el fin de consolidar el turismo como un objeto de estudio concienzudo y serio. De igual forma, el turismo como disciplina se ha convertido en una necesidad para las empresas del sector que requieren profesionales capaces de afrontar los retos de esta actividad económica, aun cuando el enfoque del turismo como objeto de estudio sea principalmente social. Por ello, los profesionales de las carreras profesionales deben adquirir competencias académicas para gestionar y suministrar servicios de calidad (Carrera, 2018).

En materia turística, los estudiantes adquieren habilidades específicas que se deben analizar a través de los indicadores de recursos humanos (Bañuls, Rodríguez y Jiménez, 2007). Estas habilidades o competencias, en muchos casos, no responden a los requerimientos de talento humano de la realidad empresarial, por lo que resulta primordial reconocer las necesidades en recursos humanos que tienen las empresas de sector turístico y hotelero con el fin de proponer mejoras curriculares a los planes de estudio de las Instituciones de Educación Superior (IES) (Díaz, 2006). Bañuls y Casado (2011) plantean, en este sentido, que existe un desajuste educativo en los planes de estudio debido en gran parte a una desarticulación entre la academia y los requerimientos laborales.

El turismo es una actividad que requiere una mano de obra especializada y competencias específicas (Bañuls 2009); razón por la cual la academia debe estar en un constante cuestionamiento acerca de las competencias profesionales que deben alcanzar los estudiantes, de manera que los egresados tengan un mejor acceso al empleo profesional (Leguizamón, 2016; Santana, 2005).

En Colombia la formación en instituciones de educación superior está a cargo de la vigilancia y supervisión del Ministerio de Educación Nacional (MEN), allí se derivan una serie de niveles educativos establecidos en pregrados y posgrados. Los pregrados se clasifican en técnicos, tecnólogos y profesionales y posgrados en especializaciones, maestrías y doctorados (MEN, 2010). Es importante hacer hincapié en que esta investigación solo tiene en cuenta las competencias de los programas profesionales, no se tuvo en cuenta los niveles técnicos, tecnólogos o de posgrado.

Teniendo en cuenta, lo anteriormente planteado este articulo presenta la relación existente entre las competencias profesionales que desarrollan los programas profesionales de hotelería y turismo y las necesidades de capital humano que tienen los empresarios del sector turístico y hotelero en la ciudad de Bogotá. Es importante hacer hincapié en que esta investigación solo tiene en cuenta las características de los empleados de nivel profesional en las empresas del sector turístico y hotelero. Por lo cual no se tuvo en cuenta los profesionales que son independientes o empresarios.

Como ya se mencionó, el turismo es un área de estudio multidisciplinar, conformada por diversas las áreas del conocimiento; las cuales se orientan de acuerdo con los intereses del investigador (Panosso, 2007; Nechar, 2007; Korstanje, 2011). Estos enfoques del turismo pueden ser desde lo ambiental, lo social, lo cultural y lo económico (Beni, 2019). No obstante, en materia de educación, los programas en turismo son recientes, y a pesar de tener un 
componente multidisciplinar, presentan una clara orientación empresarial (López y López, 2004). Por esta razón, es importante que los egresados, una vez obtengan el título profesional de turismo, precisen una especialización o factor diferencial, con el fin de tener graduados del turismo especializados, sin dejar a un lado la formación integral (Wallingre, 2011).

En varios casos para algunos autores trabajar en el sector turístico supone la ocupación de cargos operativos como recepcionista, mesero, entre otros (Dreher, Carrion y da Silveira, 2013). Esta última idea es una percepción errónea que muchos estudios tienen de la disciplina turística. Por ello, es importante aportar con investigaciones científicas, la necesidad y variedad que tiene el turismo en diferentes niveles, no solo a nivel profesional, sino a nivel técnico y tecnológico, de manera que se pueda atender a la demanda de diferentes perfiles en el sector. Sustentado en la actual coyuntura, donde el capital humano se establece como un factor estratégico de competitividad turística, debido al gran crecimiento de esta actividad (Bañuls, Rodríguez y Jiménez, 2007).

Es importante elucidar que en ocasiones el turismo no es tomado como un objeto serio de estudio, lo cual no es exclusivo de los estudiosos del turismo, sino también de los egresados de las ciencias sociales cuando se comparan con profesionales de las ciencias exactas (Gómez, 2010). Estas afirmaciones son producto de estudiosos que consideran falta de seriedad en los estudios turísticos, esto se puede controvertir, debido al aumento de investigaciones científicas del sector. Por ello, se deben realizar más estudios en materia turística y educativa, con el fin tener en cuenta las necesidades actuales del sector.

La educación del turismo debe estar conectada con las necesidades de la sociedad, así como, con la formación de profesionales capaces de actuar como educadores (Leme, 2009). Esto es imprescindible para garantizar la calidad en la educación profesional del sector. Del mismo modo, la formación docente en las carreras de licenciaturas en turismo debe aportar al desarrollo académico e investigativo de los estudiantes (Cotelo, Riol y Romero 2018). De igual forma, se deben propender acciones que permitan la profesionalización de licenciados en materia turística; debido a que en Colombia aún no hay carreras profesionales direccionadas hacia la formación de maestros en turismo.

\section{Revisión de la literatura sobre diseños curriculares en turismo}

Las competencias en la educación deben de orientarse de acuerdo con el nivel de formación; es decir según la oferta académica. De acuerdo con McClelland, citado por Yaníz (2008, p. 2), las competencias son aquellos "conocimientos, actitudes y habilidades necesarias para desempeñar una ocupación dada". Esta definición se orienta hacia el proceso de enseñanza en las IES; para la gestión de un currículo por competencias es imprescindible que el proceso de enseñanza tenga una fuerte estructura pedagógica, filosófica y epistemológica (Martínez, 2008). La cual debe estar sustentada no solo en quienes lo diseñan, sino también en quienes imparten la educación.

Según Maura (2002), una competencia es un grupo de habilidades de un individuo con relación a una buena práctica en un puesto de trabajo. No obstante, otros autores proponen la competencia como las cualidades y capacidades que son dispuestas por el individuo, a la hora de manifestar sus habilidades en un contexto específico (Gámez, 2005). Formar 
competencias profesionales según McClelland y Mertens (citado por Maura y Tirados 2008), es una de las metas de las IES en actualidad. De acuerdo con lo anterior las competencias articulan una serie de elementos que deben tener una integración, teniendo en cuenta que el aprendizaje adquirido se convierte en el vehículo para pasar de la teoría a la praxis.

Según Pedreño citado por (Hernández et al., 2010), las carreras de turismo se consolidan en Italia en los años veinte, en Alemania y Reino Unido en los años treinta y en España en los años noventa. En Colombia es apenas en 1976, cuando se constituye el primer programa de turismo (Universidad Externado de Colombia, 2014). Lo anterior evidencia la necesidad de establecer planes de estudio que permitan la construcción de la identidad turística en el país (López y Chávez, 2017). Por ello, la enseñanza en esta área se impone como una fuerza de cambio y busca nuevas propuestas de formación (Leme y Rejowski, 2011).

Los programas de educación profesional en turismo requieren diseños curriculares que integren competencias transversales y nuevas habilidades que obedezcan a las nacientes tendencias del sector; por ello, surge la necesidad de plantear estrategias que permitan renovar el aprendizaje de los estudiantes y mejorar la incursión de ellos en el mercado laboral (Cárdenas, Pulido y Carrillo, 2016). Dado que el turismo es una disciplina compleja y dialéctica, sus múltiples modalidades deben ser estudiadas desde un enfoque integral donde se interconecten los niveles del sujeto y el objeto (Campodónico y Chalar, 2011). El turismo tiene un vínculo potencial para hacer del conocimiento producido sobre la ciencia y este fenómeno un objeto de estudio, esta área de conocimiento debe tener más en estrategias didácticas interdisciplinares de investigación que permitan plantear discursos que le aporten a la disciplina turística (Nechar y Netto, 2011).

Como lo estipula el Ministerio de Comercio Industria y Turismo - MinCIT citado por Chaparro y Machado (2016, p. 50), "los contenidos turísticos y, en general, el uso creativo del tiempo libre son un eje trasversal de contenidos de todas las asignaturas, como eje articulador de las diferentes propuestas curriculares". Los componentes transversales en turismo deben estar alineados con el enfoque disciplinar de la carrera y con los objetivos del plan de estudios, de forma que las asignaturas transversales permitan complementar las temáticas disciplinares de la carrera.

Es necesario plantear los elementos del currículo en la estructura del plan de estudios, donde la interdisciplinariedad sea el medio para el equilibrio del plan de estudios (Méndez, Sierra y Mañana, 2013). En ocasiones las disciplinas que conforman el turismo como campo de estudios tienden a tener una parcialidad hacia el enfoque temático del estudio, logrando así que se aleje la posibilidad de alcanzar un enfoque holístico del turismo. Del mismo modo, los sujetos de aprendizaje deben estudiar distintas temáticas; estas principales áreas de estudio, como se mencionó anteriormente, están compuestas por los enfoques ambientales, económicos y socioculturales.

Las ciencias naturales aportan elementos necesarios para el desarrollo del turismo en las dimensiones ambientales (Chaparro y Machado, 2016). Por ello, la educación ambiental incorporada a la dinámica turística, contribuye para que los profesionales del sector comprendan los impactos del turismo en el medio ambiente. Esto es permite que los 
profesionales puedan actuar como educadores ambientales en los procesos de turismo (Leme, 2009). Este enfoque del turismo requiere de profesionales expertos en los aspectos ambientales, con el fin de planear y gestionar la capacidad de carga del turismo en el territorio y poder afrontar la gestión de nuevos productos turísticos sostenibles y responsables como los relacionados con el turismo indígena o de pueblos originarios, con grandes potencialidades de desarrollo en Colombia, pero que exige de un tratamiento muy diferenciado y singular (Rivera, Osuna y Rodríguez, 2017).

El turismo desde la perspectiva económica incorpora la importancia de esta actividad para el desarrollo de las naciones y los destinos turísticos. Para ello, se analizan las empresas turísticas, así como: la oferta, demanda, el impacto de esta actividad en la balanza comercial, las divisas y otros indicadores macroeconómicos que están ligados a las actividades turísticas (Mochón, 2008). El enfoque económico permite que los estudiantes adquieran conocimiento, para comprender las dinámicas económicas desde un enfoque cuantitativo.

El turismo tiene en cuenta diversas áreas de estudio, entre las que se distinguen las administrativas y las relacionadas con otras ciencias humanas. Es en estas últimas donde el turismo y la educación presentan una relación bastante significativa, teniendo en cuenta que son actividades propias del humanismo, por lo que implica ser un objeto de estudio interdisciplinario (Salcedo y Suárez, 2005). De igual forma, los aspectos sociales del turismo están relacionados con la geografía, antropología, psicología y demás ciencias que aportan al desarrollo integral del turismo, desde una perspectiva humana.

Existe una gran oferta formativa en turismo; sin embargo, son pocas las IES que la tienen bien estructurada como una disciplina de estudio. Además, hay una gran carencia de ofertas de posgrados y programas que promuevan el desarrollo académico del turismo en el ámbito posgradual (Pimentel, de Carvalho y de Oliveira, 2017). A este déficit de posgrados se puede argumentar que es debido a que la disciplina del turismo está en una fase de consolidación, razón por la cual es apenas normal que existan pocas ofertas de este nivel. Por ello, se deben establecer los aspectos clave en la educación profesional del turismo, y así fortalecer esta disciplina académica.

\section{Metodología}

Esta investigación está basada en un enfoque cualitativo, ya que permite describir conceptos y establecer las relaciones entre nociones para reconocer las causas de los eventos sociales (Hernández, Fernández y Baptista, 2010; Izcara, 2014). Adicional, esta investigación es ideal para abordar las realidades educativas, ya que no solo pretende obtener datos cualitativos, sino también tener una visión más precisa de estos datos (Pérez, 2011).

La presente investigación es de nivel descriptivo (Arias, 2015), porque explica la relación entre dos categorías. Por un lado, describe las competencias educativas plasmadas en los planes de estudio, que dan como resultado, competencias profesionales en estudiantes de carreras universitarias en turismo. Por otro lado, aborda las necesidades de talento humano en las empresas que ofertan servicios turísticos en Bogotá D.C. Esto permite identificar la relación de los conocimientos adquiridos por los egresados con las necesidades reales de los empresarios turísticos. Para el diseño de los instrumentos de investigación se realizó una 
categorización, donde se compilaron los elementos o aspectos con características comunes que se relacionan entre sí. Esto permitió establecer una clasificación y agrupar elementos en común para el desarrollo metodológico del trabajo de campo.

Para el diseño, y análisis de los resultados se establecieron categorías de análisis, las cuales tuvieron en cuenta los aspectos de la oferta educativa de nivel profesional. Para ello, se reconocieron las competencias educativas plasmadas en los planes de estudio vigentes al 2019, de todos los programas profesionales en materia turística, para ese entonces habían 13 IES ofertando tales programas. Posteriormente, de seleccionaron los programas de las siguientes instituciones CUN, UNIMINUTO y UNIAGUSTINIANA. Allí se realizó una descripción de cuáles son los ejes curriculares, bajo los cuales se fundamentan los programas disciplinares en turismo. Para esta fase de la investigación se realizaron entrevistas estructuradas y semiestructuradas con los directores académicos de los programas, esto permite conocer la opinión de ellos y establecer las competencias académicas de los programas. Principalmente en los aspectos relacionados con el perfil profesional de los egresados, así como sus principales enfoques teóricos.

Para el sector empresarial se indago acerca de las necesidades de talento humano de las empresas que ofertan servicios turísticos en la ciudad de Bogotá. Los resultados permitieron identificar las principales necesidades en los cargos profesionales. Para ello, se realizó una selección aleatoria de 20 Prestadores de Servicios Turísticos (PST), allí se realizaron entrevistas a las personas con decisión en la contratación de los cargos profesionales. Se entrevistaron a los directores de recursos humanos y contratación, quienes indicaron las necesidades laborales en el ámbito profesional. Finalmente se realizó una búsqueda de los programas profesionales en la ciudad de Bogotá D.C. De acuerdo con la información del SNIES, sistema del MEN, para los programas académicos de las IES en Colombia, los criterios de búsqueda se relacionan en la siguiente tabla.

Tabla 1. Criterios para la búsqueda de programas profesionales en turismo en Bogotá

\begin{tabular}{ll}
\hline Criterios de búsqueda & Información que se utilizó para la consulta \\
\hline Nombre del programa & $\begin{array}{l}\text { Se realizaron búsquedas con las palabras: Turismo, } \\
\text { Hotelería, Turística y Hotelera. }\end{array}$ \\
\hline Estado de la institución & Activa \\
\hline Estado del programa & Activo \\
\hline $\begin{array}{l}\text { Municipio de oferta del } \\
\text { programa }\end{array}$ & Bogotá D.C. \\
\hline Nivel Académico: & Pregrado \\
\hline Nivel de Formación & Universitario \\
\hline Metodología & Distancia, Presencial y Virtual \\
\hline
\end{tabular}

Fuente: Elaboración propia a partir de MEN (2019). 


\section{Resultados}

A continuación, se describen los resultados de las entrevistas, por un lado, con los directores académicos y, por otro, con los encargados de la contratación en los PST; quienes establecen los lineamientos para la contratación de los cargos profesionales. Los resultados se muestran de forma general buscando así por medio de las categorías de análisis, reconocer la visión que se tiene en general de forma académica y empresarial. Como se puede apreciar en Bogotá D, C., los programas se ofertan en diferentes modalidades de estudio, El $77 \%$ de los programas están estructurados bajo la modalidad presencial. El 8\% bajo la modalidad a distancia. Por último, el $15 \%$ de los programas bajo la metodología virtual.

Tabla 2. Oferta de programas profesionales en hotelería, turismo en Bogotá D.C. a junio del 2019.

\begin{tabular}{|c|c|c|c|}
\hline Nombre de la IES & Programa & Modalidad & Área de Conocimiento \\
\hline $\begin{array}{lr}\text { Universidad } & \text { Colegio } \\
\text { Mayor } & \text { de } \\
\text { Cundinamarca } & \end{array}$ & Turismo & Presencial & $\begin{array}{l}\text { Economía, } \\
\text { Administración, } \\
\text { Contaduría y afines }\end{array}$ \\
\hline $\begin{array}{l}\text { Universidad Antonio } \\
\text { Nariño }\end{array}$ & $\begin{array}{l}\text { Hotelería y } \\
\text { Turismo Ecológico }\end{array}$ & Presencial & $\begin{array}{l}\text { Economía, } \\
\text { Administración, } \\
\text { Contaduría y afines }\end{array}$ \\
\hline $\begin{array}{l}\text { Universitaria } \\
\text { Agustiniana- } \\
\text { UNIAGUSTIANA }\end{array}$ & $\begin{array}{l}\text { Hotelería } \\
\text { Turismo }\end{array}$ & Presencial & $\begin{array}{l}\text { Economía, } \\
\text { Administración, } \\
\text { Contaduría y afines }\end{array}$ \\
\hline $\begin{array}{l}\text { Fundación } \\
\text { Universitaria para el } \\
\text { Desarrollo Humano - } \\
\text { UNINPAHU }\end{array}$ & $\begin{array}{l}\text { Profesional } \\
\text { Turismo } \\
\text { Gastronomía }\end{array}$ & Presencial & $\begin{array}{l}\text { Economía, } \\
\text { Administración, } \\
\text { Contaduría y afines }\end{array}$ \\
\hline $\begin{array}{l}\text { Universidad Externado } \\
\text { De Colombia }\end{array}$ & $\begin{array}{l}\text { Administración De } \\
\text { Empresas } \\
\text { Turísticas } \\
\text { Hoteleras }\end{array}$ & Presencial & $\begin{array}{l}\text { Economía, } \\
\text { Administración, } \\
\text { Contaduría y afines }\end{array}$ \\
\hline $\begin{array}{l}\text { Universidad Manuela } \\
\text { Beltrán - UMB }\end{array}$ & $\begin{array}{l}\text { Administración } \\
\text { Turística y Hotelera }\end{array}$ & Virtual & $\begin{array}{l}\text { Economía, } \\
\text { Administración, } \\
\text { Contaduría y afines }\end{array}$ \\
\hline
\end{tabular}




\begin{tabular}{|c|c|c|c|}
\hline $\begin{array}{l}\text { Universidad Antonio } \\
\text { Nariño }\end{array}$ & $\begin{array}{lr}\text { Administración De } \\
\text { Empresas } & \\
\text { Turísticas } & \text { Por } \\
\text { Ciclos - } & \text { Ciclo } \\
\text { Profesional } & \end{array}$ & Virtual & $\begin{array}{l}\text { Economía, } \\
\text { Administración, } \\
\text { Contaduría y afines }\end{array}$ \\
\hline $\begin{array}{l}\text { Fundación } \\
\text { Universitaria } \\
\text { Libertadores }\end{array}$ & $\begin{array}{l}\text { Administración } \\
\text { Turística y Hotelera }\end{array}$ & Presencial & $\begin{array}{l}\text { Economía, } \\
\text { Administración, } \\
\text { Contaduría y afines }\end{array}$ \\
\hline $\begin{array}{l}\text { Fundación } \\
\text { Universitaria } \\
\text { Empresarial de la } \\
\text { Cámara de Comercio de } \\
\text { Bogotá }\end{array}$ & $\begin{array}{l}\text { Administración } \\
\text { Turística }\end{array}$ & Presencial & $\begin{array}{l}\text { Economía, } \\
\text { Administración, } \\
\text { Contaduría y afines }\end{array}$ \\
\hline $\begin{array}{l}\text { Corporación } \\
\text { Universitaria Minuto } \\
\text { De Dios } \\
\text { UNIMINUTO }\end{array}$ & $\begin{array}{ll}\text { Administración } & \text { de } \\
\text { Empresas } & \\
\text { Turísticas } & \text { y } \\
\text { Hoteleras } & \end{array}$ & A distancia & $\begin{array}{l}\text { Economía, } \\
\text { Administración, } \\
\text { Contaduría y afines }\end{array}$ \\
\hline $\begin{array}{l}\text { Corporación } \\
\text { Universitaria UNITEC }\end{array}$ & $\begin{array}{ll}\text { Administración } & \text { de } \\
\text { Empresas } & \\
\text { Turísticas } & \text { y } \\
\text { Hoteleras } & \end{array}$ & Presencial & $\begin{array}{l}\text { Economía, } \\
\text { Administración, } \\
\text { Contaduría y afines }\end{array}$ \\
\hline $\begin{array}{l}\text { Corporación Unificada } \\
\text { Nacional De Educación } \\
\text { Superior CUN- }\end{array}$ & $\begin{array}{l}\text { Administración } \\
\text { Turística y Hotelera }\end{array}$ & Presencial & $\begin{array}{l}\text { Economía, } \\
\text { Administración, } \\
\text { Contaduría y afines }\end{array}$ \\
\hline $\begin{array}{l}\text { Fundación } \\
\text { Universitaria Cafam - } \\
\text { UNICAFAM }\end{array}$ & $\begin{array}{l}\text { Administración } \\
\text { Turística y Hotelera }\end{array}$ & Presencial & $\begin{array}{l}\text { Economía, } \\
\text { Administración, } \\
\text { Contaduría y afines }\end{array}$ \\
\hline
\end{tabular}

Elaboración propia a partir del MEN (2019)

Los programas mencionados en la tabla 2 tienen una oferta activa, Con un componente cenrado en las ciencias administrativas. El único programa que tiene la denominación de turismo es la Universidad Colegio Mayor de Cundinamarca, del mismo modo este es el único programa ofertado por una universidad pública. Por otro lado, en el programa de Hotelería y Turismo de la Uniagustiniana, luego de una revisión al plan de estudios, se evidencia que tiene un gran componente de las ciencias administrativas al igual que en el programa Hotelería y Turismo Ecológico de la Universidad Antonio Nariño.

\subsection{Directores Académicos}

Luego de realizar las entrevistas a los directores de las carreras se evidencia la estructura de los programas académicos, sus principales fortalezas y debilidades en los aspectos curriculares. A continuación, se muestran las respuestas de los directores 
encuestados. Estas entrevistas recopilan información, de acuerdo con la opinión de los directores, en este apartado se exponen las respuestas en común, se analiza la información de forma general y no desde las particularidades de los entrevistados.

Tabla 3. Descripción curricular en los programas profesionales en turismo.

Objetivos de aprendizaje El objetivo de los programas es formar profesionales integrales de los programas con una visión para organizaciones modernas del sector profesionales de hotelería y turístico, hotelero, gastronómico y la prestación de servicios turismo especializados que se ajusten a las necesidades del entorno con calidad, eficiencia, liderazgo y pertinencia. Del mismo modo, brindar aspectos importantes como la gerencia e innovación en el desarrollo del sector y dentro de la estructura, el proceso de principios éticos y morales orientados hacia la formación fundamentalmente. Esto con el fin de que los alumnos tengan la capacidad de administrar estas organizaciones y que puedan liderar la innovación al interior del sector en hoteles, restaurantes y catering.

Perfil del egresado El egresado está en capacidad de diseñar, planear y producir proyectos y artículos del sector turístico, hotelero y gastronómico acorde con las normas profesionales las necesidades del sector productivo. Puede asumir roles estratégicos en las organizaciones de la industria y ejercer funciones de dirección, supervisar líneas de producción, y principalmente, ser un empresario del sector. Debe tener una formación integral inclinada a los valores como tal para el desarrollo de la actividad turística, en la gerencia, en la organización, en el avance de productos turísticos y en especial orientados a los valores y al ser una persona consciente de su realidad, de su patrimonio y del servicio a la comunidad. Incluso, no necesariamente tienen que ser del sector turístico y hotelero, ellos podrán liderar diferentes áreas, como el área gestión humana de un hotel, también podrían liderarlo. para efectuarla, en dado caso, intraemprendimiento en sus empresas o crear nuevas organizaciones.

Ejes curriculares de los Los ejes curriculares del programa se dividen en fundamentos programas específicos o ejes disciplinares. Los ejes relacionados con las humanidades, matemáticas, idiomas y el emprendimiento. En el componente especifico, están los temas de la línea turística y hotelera, teorización académica inicial del turismo, la etimología, hasta que se llega a la administración. La línea hotelera tiene a su vez una línea marcada en la operación, el desarrollo de la actividad también de los establecimientos 
hoteleros en sus procesos de gestión, auditoría y las áreas operativas. ambién un componente gastronómico orientado a aplicar los principios básicos de tal área.

Competencias de los Las competencias que adquieren los egresados están basadas de egresados manera específica en el hacer y en el saber. Se pueden desempeñar en el medio de producción, administrador, diseñador de menús. Las competencias profesionales del estudiante están orientadas a su perfil ocupacional, que está centrado en desarrollar unas competencias orientadas al: liderazgo en la actividad turística, gerencia de empresas turísticas y hoteleras, gestión responsable de los recursos, al desarrollo del emprendimiento, asesoría y planeación de proyectos turísticos, innovación de productos y servicios turísticos, a ejercer la consultoría, y a construir propuestas y proyectos de investigación turística. Además, de competencias de emprendimiento y no solo de emprender pensando en crear empresas sino hacer intraemprendimiento, entendido como innovación en su mismo puesto de trabajo.

Perfil de los docentes del Los docentes que integran los programas están organizados de programa y su formación manera interdisciplinaria, lo que quiere decir que hay ingenieros disciplinar de alimentos, administradores de empresas, administradores hoteleros y turísticos, chefs, geógrafos y antropólogos. Se trata en lo posible de tener dos líneas de trabajo, la línea turística y la línea hotelera; esto con el fin de formar profesionales integrales. Adicional, la gran mayoría titulados en especialidades o en carreras relacionadas con el turismo y la hostelería como pregrado y sus especializaciones están centradas en el componente administrativo, disciplinar, cultural y práctico patrimonial dependiendo de las necesidades que se tengan. Es importante resaltar, que encontrar profesionales en la rama turística y hotelera y que logren hacer sus estudios de posgrado en estas especialidades es un proceso complicado, ya que en Colombia no hay una oferta muy amplia de maestrías ni de doctorados en el área. Sin embargo, hay profesores que tienen maestrías en el área de hotelería y turismo.

Los docentes para asignaturas de las ciencias sociales son más comunes. Los profesores disciplinares en el componente de administración de empresas, algunos tienen posgrados, orientados principalmente en la especialidad de la gerencia. En síntesis, los administradores de empresas y los humanistas son los que conforman en su mayoría la planta docente. 


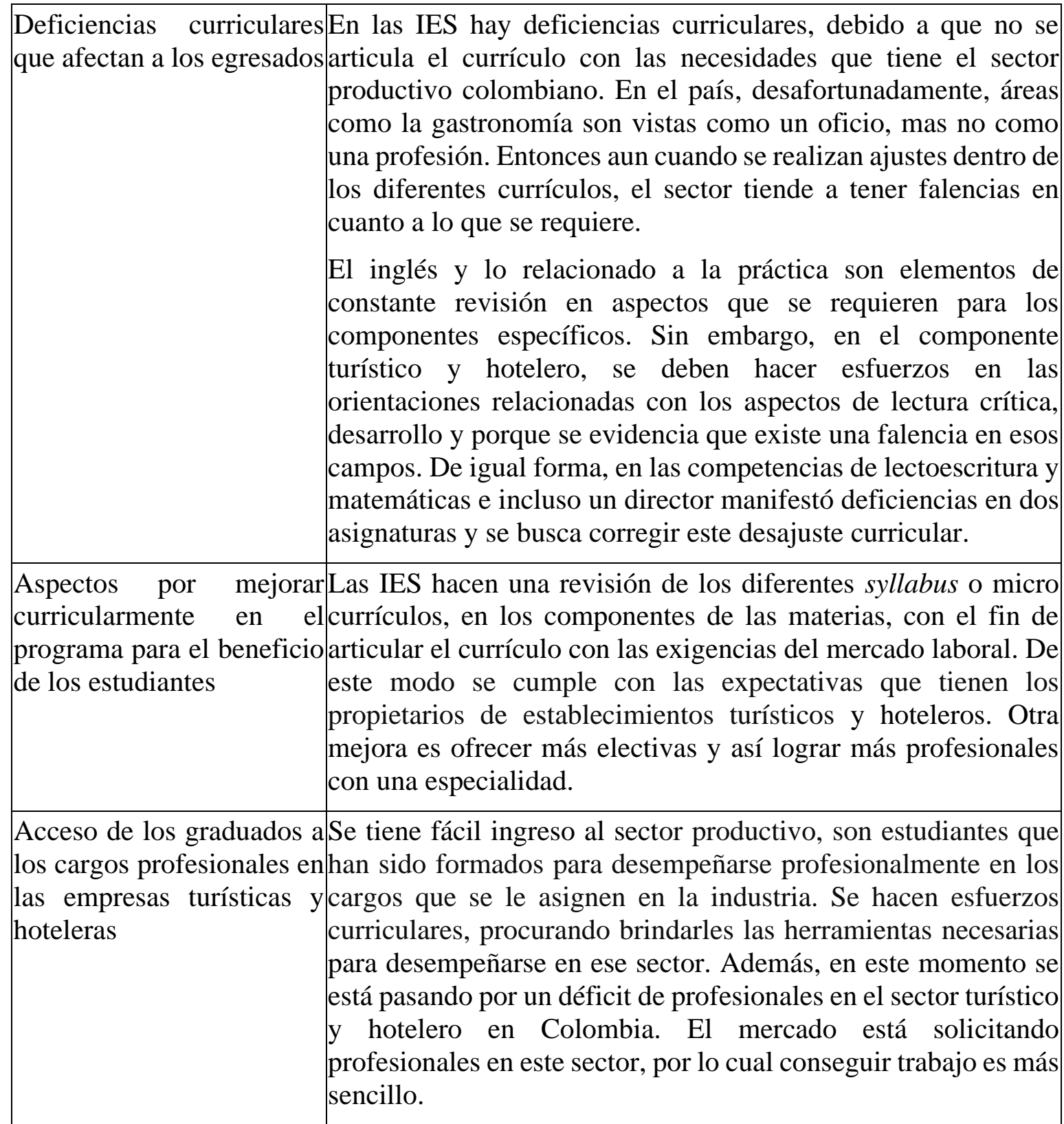

Fuente: Elaboración propia

De acuerdo con lo anterior, se puede evidenciar el punto de vista de los directores acerca del estado curricular actual de los programas que ellos tienen a su cargo. Esto permite hacer un proceso de autoevaluación con el fin de describir cuál es el estado actual de los procesos curriculares en turismo en la ciudad de Bogotá D.C., en materia profesional. Es imperativo aclarar que todos los directores académicos que participaron en esta investigación tienen título de maestría, lo que indica que son personas que están en la capacidad para autoevaluar el programa. 
Los directores que se entrevistaron llevan 2 años o más en el programa, lo cual demuestra un criterio para reconocer las debilidades y fortalezas curriculares. Los directores en su mayoría han realizado procesos de renovación curricular de acuerdo con los requerimientos establecidos por el MEN. Todos ellos tienen una gran experiencia y han sido directores en otros programas académicos, lo cual evidencia una amplia trayectoria en procesos educativos en IES. Los directores indican que los programas a su cargo son sobresalientes, sin embargo, exponen que los planes de estudio están sujetos a mejoras.

Es importante hacer hincapié en que este estudio se logra luego de recopilar entrevistas que recogen diversas opiniones y puntos de vista de los agentes implicados. Esto en cierto modo tiene ciertas limitaciones, ya que algunos directivos pueden omitir aspectos negativos, o ciertas limitaciones por temor a exponer las deficiencias en su oferta educativa. De igual forma, indican que sus planes de estudios integran al sector empresarial, pero se evidencia que no hay muchos detalles acerca de estos acercamientos. Se podría aseverar que la información allí plasmada puede darse de forma muy subjetiva. Es importante que las IES tengan un mayor vínculo con los empresarios para reconocer las necesidades del sector y de este modo ajustar los planes de estudio de acuerdo con las necesidades de los PST.

\subsection{Directores de recursos humanos}

Este epígrafe esboza las respuestas de las entrevistas realizadas a los directores de recursos humanos o quienes tienen decisión en la contratación de los PST en la ciudad de Bogotá D.C. Estas entrevistas recopilan la información, de acuerdo a las respuestas en común. Esto con el fin de analizar cuál es el punto de vista general del sector empresarial turístico.

Tabla 4. Entrevista a los directores de recursos humanos

\begin{tabular}{|l|l|}
\hline $\begin{array}{l}\text { Principales } \\
\text { necesidades } \\
\text { laborales para los } \\
\text { cargos profesionales }\end{array}$ & $\begin{array}{l}\text { En el caso de agencias de viajes: manejo de sistemas de } \\
\text { distribución, principalmente Sabre y Amadeus. Uso del sistema } \\
\text { Zeus en el caso de los hoteles. Manejo intermedio del paquete de } \\
\text { office en especial Excel. Manejo del idioma inglés y de preferencia } \\
\text { una tercera lengua, conocimiento de geografía turística. Servicio al } \\
\text { cliente, manejo de personal, capacidad de resolver problemas de } \\
\text { servicio al cliente y excelentes relaciones interpersonales. } \\
\text { Capacidad de direccionar proyectos e innovación. Constante } \\
\text { actualización y adaptación al mercado. }\end{array}$ \\
\hline $\begin{array}{l}\text { Competencias } \\
\text { laborales en } \\
\text { empleados } \\
\text { profesionales }\end{array}$ & $\begin{array}{l}\text { Tener capacidad de trabajar bajo presión, manejar situaciones } \\
\text { difíciles de último momento. Toma de decisiones, identificar } \\
\text { debilidades y proponer acciones de mejora. Interactuar con el } \\
\text { medio con el fin de fortalecer las relaciones comerciales, trabajo en } \\
\text { equipo y liderazgo. Herramientas ofimáticas, trabajo en equipo, } \\
\text { inglés y/o un idioma más, Excel, geografía, servicio al cliente. }\end{array}$ \\
\hline
\end{tabular}




\begin{tabular}{|c|c|}
\hline $\begin{array}{l}\text { Dificultad que } \\
\text { tienen los egresados } \\
\text { en carreras } \\
\text { profesionales } \\
\text { relacionadas con el } \\
\text { turismo }\end{array}$ & $\begin{array}{l}\text { En agencias de viaje muy poco conocimiento en sistemas de } \\
\text { distribución y de reservas con los diferentes proveedores, en } \\
\text { geografía turística, en idioma inglés. No hay buenas bases de } \\
\text { prácticas y materias con poca práctica. Muchos no están en la } \\
\text { capacidad de brindar una experiencia extraordinaria al cliente más } \\
\text { allá del servicio o la atención básica. Esto es un factor diferencial } \\
\text { frente a los sistemas de reservas de internet, a las aplicaciones } \\
\text { disponibles hoy en día para suplir los servicios de turismo. }\end{array}$ \\
\hline $\begin{array}{l}\text { Fortalezas en los } \\
\text { profesionales en } \\
\text { Hotelería y Turismo }\end{array}$ & $\begin{array}{l}\text { Disposición para el aprendizaje, son propositivos, tienen grandes } \\
\text { expectativas, son apasionados, responsables, innovadores y } \\
\text { multifuncionales. Poseen una visión global que hoy en día los } \\
\text { profesionales desarrollan sobre el negocio para adelantarse a las } \\
\text { necesidades del cliente. }\end{array}$ \\
\hline $\begin{array}{l}\text { Debilidades de los } \\
\text { egresados en } \\
\text { carreras de turismo }\end{array}$ & $\begin{array}{l}\text { La debilidad más grande es la falta de experiencia, en segundo } \\
\text { lugar, falta de dominio del idioma inglés. Además, tienden a actuar } \\
\text { por instinto, sin detenerse un poco a estudiar las políticas de la } \\
\text { compañía. Esta visión global debe complementarse con la } \\
\text { capacidad de resolver situaciones difíciles con los clientes y } \\
\text { viajeros, para tomar decisiones asertivas en medio de la presión y } \\
\text { la urgencia del negocio. }\end{array}$ \\
\hline $\begin{array}{l}\text { Incentivos a los } \\
\text { empleados de los } \\
\text { niveles técnicos y } \\
\text { tecnólogos hacia la } \\
\text { profesionalización }\end{array}$ & $\begin{array}{l}\text { Se da la oportunidad de que los empleados vayan ascendiendo de } \\
\text { acuerdo con sus capacidades y experiencia, así como también con } \\
\text { su nivel de estudios. Se procura hacer negociaciones y campañas } \\
\text { de crédito con las entidades correspondientes para fines } \\
\text { estudiantiles. Estos apoyos se dan siempre y cuando alcancen una } \\
\text { curva de aprendizaje, que permita combinar horario de estudio y } \\
\text { trabajo, con madurez, responsabilidad de lo que hacen. De igual } \\
\text { forma, las compañías brindan la posibilidad a los practicantes de } \\
\text { continuar sus estudios y desarrollar sus prácticas como } \\
\text { profesionales dentro de la compañía, con un pago por apoyo de } \\
\text { sostenimiento superior al nivel de técnico o tecnólogo. }\end{array}$ \\
\hline $\begin{array}{lr}\text { Capacitación } & \text { a los } \\
\text { empleados } & \text { que } \\
\text { ocupan } & \text { cargos } \\
\text { profesionales } & \end{array}$ & $\begin{array}{l}\text { Existen programas de capacitación que se hacen con los operadores } \\
\text { turísticos, funcionarios de otros cargos con más experiencia en el } \\
\text { país, así como también conferencias en distintos campos como lo } \\
\text { son: liderazgo, servicio al cliente etc. Además, es obligatorio en } \\
\text { los procesos de inducción, acompañamiento en la curva de } \\
\text { aprendizaje y actualización de conocimientos. Incluso, una } \\
\text { compañía indicó que se realiza un proceso de entrenamiento al } \\
\text { momento de ingresar a la compañía como profesional, el cual dura } \\
9 \text { semanas y adicional se brinda el acompañamiento de mentores } \\
\text { durante los siguientes meses para fortalecer los conocimientos y el }\end{array}$ \\
\hline
\end{tabular}




\begin{tabular}{|l|l|}
\hline & $\begin{array}{l}\text { afianzamiento con la cultura, herramientas y procesos internos para } \\
\text { brindar el mejor servicio a los clientes. }\end{array}$ \\
& \\
$\begin{array}{l}\text { Sugerencias a las } \\
\text { universidades para } \\
\text { mejorar el recurso } \\
\text { humano en las } \\
\begin{array}{l}\text { Empresas } \\
\text { turismo del }\end{array}\end{array}$ & $\begin{array}{l}\text { Incentivar un poco más el dominio de una segunda lengua, motivar } \\
\text { en rentabilidad, sin descuidar la parte humana. Habilitar } \\
\text { convenios de prácticas profesionales enfocadas en orientar al } \\
\text { profesional a ser integral. Además, se deben complementar los } \\
\text { conocimientos técnicos con el desarrollo de otros idiomas. }\end{array}$ \\
\hline $\begin{array}{l}\text { Disciplinas afines } \\
\text { laboralmente } \\
\text { profesional al } \\
\text { turismo en }\end{array}$ & $\begin{array}{l}\text { Administradores de empresas, en general con carreras de las } \\
\text { ciencias administrativas o empresariales. }\end{array}$ \\
\hline
\end{tabular}

Fuente: Elaboración propia

Los empresarios indican que los ejes curriculares que ofertan las IES en programas profesionales están relacionados con las necesidades de las empresas. Es decir, que hay una relación entre los objetivos de enseñanza y los requerimientos de los empresarios. Esto demuestra que en los aspectos teóricos existe una relación de lo que tienen los programas académicos con lo que requiere el sector laboral. Sin embargo, en aspectos como las habilidades blandas, y la práctica profesional las IES tienen una oportunidad de mejora. Se evidencia que hay una relación entre los aspectos teóricos que ofertan las IES, no obstante, se evidencia un déficit de praxis. Es decir que hay aspectos teóricos que las instituciones deberían incrementar, teniendo en cuenta que el turismo requiere de grandes habilidades operativas.

\section{Conclusiones}

En materia educativa el turismo es una área de estudios compleja, debido a su naturaleza multidisciplinar (Korstanje, 2011; Nechar, 2007). Además, no son unánimes las construcciones curriculares en esta materia, y las particularidades de cada uno de los programas presentan grandes diferencias. No obstante, se destaca el componente administrativo en las denominaciones de los programas académicos. También se resalta una buena autoevaluación en materia curricular, de acuerdo con las entrevistas realizadas; no obstante, esto puede ser por una sobrevaloración, debido a que los directores autoevalúan el programa desde su gestión. Por otro lado, los empresarios del sector determinan que los planes de estudio o el currículo se encuentran en buena forma, es decir aprueban desde el punto de visto teórico, pero no desde la praxis. Adicionalmente, indican que los egresados de 
los programas de turismo carecen de ciertas competencias, que requieren las empresas para ocupar las vacantes del personal profesional.

El perfil de formación de los profesionales del sector tiene una gran orientación hacia la creación de empresa, dirección y formulación de proyectos, dado el gran enfoque administrativo que se da en los objetivos de educación de los programas profesionales. Todas las IES ofertan sus planes de estudio con enfoque de transversalidad (ciencias empresariales) y asignaturas o componentes específicos (hotelería y turismo). Como es de saberse, el turismo es un área polisémica y es una disciplina que apenas se está consolidando (Jafari, 2005). Por ello, los programas presentan discrepancias, se deben revisar las orientaciones, e incluso analizar muy bien las denominaciones de los programas.

Las empresas del sector brindan un fácil acceso a los cargos operativos de los "nuevos" profesionales en turismo; por lo general esta oferta laboral está dada hacia los cargos técnicos. Es evidente que la vinculación hacia el "primer empleo" está casi que garantizada. Incluso, sin la certificación como profesional, los estudiantes y/o profesionales en hotelería y turismo tienen una gran oportunidad debido al crecimiento de la industria turística en el país. Sin embargo, en la mayoría de las convocatorias laborales ellos compiten con administradores de empresas y otras carreras orientadas desde las ciencias económicas. Es importante desarrollar especialidades en los planes de estudio de acuerdo con las necesidades específicas de los PST.

El turismo a pesar de ser una disciplina reciente es un área de estudios con gran proyección educativa y las IES ven con buenos ojos el trabajo que se ha venido desarrollando en sus planes de estudio. Aunque, son conscientes que debe existir una mejora continua es decir una revisión curricular constante.

Por otro lado, la percepción de los empresarios con respecto al currículo y/u oferta académica de la IES, es bastante diferente. Los directores de recursos humanos o las personas encargadas de la contratación tienen un punto de vista negativo respecto a la formación de los profesionales. Argumentando como principal factor la falta de experiencia, catalogando como un exceso de discurso en las competencias teóricas y descuidando las competencias prácticas.

Es evidente que el turismo como profesión ha adquirido un papel importante, debido a las múltiples ofertas académicas profesionales que han surgido en los últimos años. Las empresas del sector turístico requieren constantemente de personal calificado para ocupar diversos cargos. En muchos casos las empresas aportan al desarrollo del talento humano, capacitando a sus empleados, de acuerdo a las características del perfil. Sin embargo, es importante destacar que no siempre se limitan a contratar profesionales en áreas de hotelería y turismo.

La demanda de empleos en este sector va a ser constante debido al gran crecimiento de la industria turística en el país (Mora y Bohórquez, 2018; Mora, 2018; Mora, 2019; Mora, Yamova y Murtuzalieva, 2019). Es por ello, que se recomienda para futuras investigaciones analizar el empleo en turismo y las necesidades de talento humano. De igual forma, también se necesita orientar las investigaciones con enfoques económicos, dado que esta investigación aborda principalmente los aspectos cualitativos de la educación del turismo. 
Es importante resaltar que el turismo dada su naturaleza multidisciplinaria, no se puede generalizar en términos académicos. Por ello, se debe lograr la consolidación de distintos enfoques del turismo, haciendo énfasis en la denominación de carreras con enfoque empresarial, ambiental y social, los cuales son de gran importancia. Esto permitirá tener una mejor clasificación y proyección de los profesionales que estudian carreras de hotelería y turismo.

Esta investigación se planteó con estudios de casos en las IES de la ciudad de Bogotá, D.C. por esta razón se deben realizar estudios con casos en otras regiones distintas al interior del país. Esto permitirá tener una concepción mayor acerca de los aspectos curriculares faltantes y las necesidades de cada ciudad o región. De igual forma, se hace énfasis que Bogotá es la ciudad con la mayor oferta educativa en turismo en el país. Por lo cual, se podría llegar a aseverar que en otras regiones del país el problema puede llegar a ser más agudo, debido a la baja oferta académica.

Los hallazgos de la presente investigación permiten a las IES adaptar las necesidades del sector empresarial a las necesidades de las empresas del sector turísticos en el país. Como principales debilidades se identifica la falta de bilingüismo y la inexperiencia o ausencia en la adquisición de competencia prácticas. En este orden de ideas teniendo en cuenta que el turismo es un área de estudios en proceso de construcción, se deben incrementar los procesos prácticos en el sector empresarial; es decir más horas de pasantías, incluso que estas sean obligatorias e incrementar el tiempo de las mismas. Debido a que es la principal debilidad según los empresarios del sector turístico y hotelero. Del mismo modo, se deben incrementar las acciones para lograr que los profesionales adquieran mayores habilidades en el bilingüismo, esto se puede lograr a través del desarrollo de la segunda lengua como requisito de grado.

En términos generales, se puede concluir que existen discrepancias entre lo que enseñan las IES y lo que requieren los PST. Por ello, se invita a las IES a incorporar al sector externo (los PST), en la planeación y las decisiones educativas que corresponden a los aspectos curriculares. Para ello, se pueden generar ofertas académicas en conjunto donde los estudiantes adquieran competencias teóricas en la academia y obtener las prácticas en los PST. En síntesis, es imperativo que las IES aumenten los esfuerzos prácticos; orientados hacia las necesidades en talento humano de los PST. Esto último, permitirá formar profesionales para desempeñarse de forma óptima los cargos profesionales de los PST del país.

\section{Referencias}

Arias, J. A. C. (2015). Ortodoxia y fisuras en el diseño y ejecución de estudios descriptivos. Revista Med, 23(1), 40-51.

Avello Martínez, R., López Fernández, R., \& Vázquez Cedeño, S. (2015). Competencias TIC de los docentes de las escuelas de hoteleria y turismo cubanas. Universidad y sociedad, 63-69. 
Bañuls, A. L., Rodríguez, A. B. R., \& Jiménez, M. S. (2007). El capital humano como factor estratégico para la competitividad del sector turístico. Cuadernos de turismo, (19), 4769. Obtenido de https://revistas.um.es/turismo/article/view/13831

Bañuls, A. L. (2009). El papel del capital humano en el sector turístico: algunas reflexiones y propuestas. Cuadernos de turismo, (24), 53-64.

Bañuls, A. L., \& Casado-Díaz, J. M. (2011). Capital humano y turismo: Rendimiento educativo, desajuste y satisfacción laboral. Estudios de economía aplicada, 29 (3), 626.

Beni, M. C. (2019). Análise estrutural do turismo. Sao Pablo: Senac.

Campodónico, R., \& Chalar, L. (2010). Turismo: una ciencia en construcción. Anais do VI Seminario de Pesquisa em turismo do MERCOSUL-Saberes no turismo: Interfaces, Caxias do Sul, 1-15.

Cárdenas-García, P. J., Pulido-Fernández, J. I., \& Carrillo-Hidalgo, I. (2016). Adquisición de competencias en el Grado de Turismo mediante el aprendizaje basado en estudios de caso. Aula Abierta, 44(1), 15-22. doi.org/10.1016/j.aula.2015.05.002

Carrera, P. (2018). Diseño del sistema académico por competencias de la Escuela de Turismo y Hospitalidad de la Pontificia Universidad Católica del Ecuador. Gestión Turística, (13), 113-127. DOI:10.4206/gest.tur.2010.n13-05

Chaparro, A. D., \& Machado, R. (2016). La formación en cultura turística en instituciones educativas: un aporte del programa "colegios amigos del turismo" en Colombia. Turismo y Sociedad, (19), 49-71. doi.org/10.18601/01207555.n19.04

Cotelo-Armenteros, S., Riol-Hernández, M., \& RomeroViamonte, J. (2018). La profesionalización docente en género: contribución al referente de género del profesorado universitario. Educación y Sociedad, 1(1), 95-107. Obtenido de http://revistas.unica.cu/index.php/edusoc/article/view/1028.

Díaz Barriga, Á. (2006). La educación en valores: Avatares del currículum formal, oculto y los temas transversales. Revista electrónica de investigación educativa, 8(1), 1-15. Obtenido de http://www.scielo.org.mx/scielo.php?pid=S1607$40412006000100001 \&$ script=sci_abstract\&tlng=en.

Dreher, M. T., Carrion, R. D. S. M., \& da Silveira, A. P. K. (2013). Ocupación en el sector turístico: inclusión social y prejuicios. Cuadernos de Turismo, (32), 281-294. Obtenido de https://revistas.um.es/turismo/article/view/177571

Fayos-Solá, E. (1997). Educación y formación en la Nueva Era del Turismo: la visión de la OMT. El capital humano en la industria turística del siglo XXI, 59-72.

Gámez, A. N. (2005). Propuestas conceptuales en torno a la competencia profesional. Revista de educación, 337, 213-234. Obtenido de http://www.revistaeducacion.educacion.es/re337/re337_11.pdf. 
Gómez Nieves, S. (2010). La experiencia de un diseño curricular en turismo basado en un modelo por competencias profesionales. Estudios y perspectivas en turismo, 19 (1), 139-156. Obtenido de https://www.redalyc.org/pdf/1807/180713899008.pdf.

Hernández, C. C., Martín, C. A., Jiménez, A. R., Domínguez, C. S., \& Bermúdez, I. V. (2010). La formación en turismo en España: pasado, presente y futuro en el nuevo Espacio Europeo de Educación Superior. Cuadernos de Turismo, (25), 45-67. Obtenido de https://revistas.um.es/turismo/article/view/109571.

Hernández, R., Fernández, C., \& Baptista, M. D. P. (2010). Los métodos mixtos. Metodología de la investigación. 5th ed. México: Mc Graw Hill, 544-601.

Herrera, S. R. G. (2018). La práctica preprofesional en el currículum de la carrera Turismo. Revista Conrado, 14(64), 40-45.

Izcara Palacios, S. P. (2014). Manual de investigación cualitativa. México: Fontamara.

Jafari, J. (2005). El turismo como disciplina cientifica. Política y Sociedad, (42), 39-56.

Korstanje, M. E. (2011). Mitología y turismo. La exégesis como interpretación hermenéutica. Estudios y perspectivas en turismo, 20 (6). 1258-1280. Obtenido de https://www.redalyc.org/pdf/1807/180722700002.pdf.

Leguizamón, M. (2016). Empleo formal e informal en el sector turístico en Colombia. Turismo y Sociedad, (18), 179-187. doi.org/10.18601/01207555.n18.10

Leme, F. B. M. (2009). Educación ambiental y turismo: una formación holística, interdisciplinaria y de futuros educadores. Estudios y perspectivas en turismo, 18 (1), 92-106. Obtenido de https://www.redalyc.org/articulo.oa?id=1807/180714242006

Leme Sogayar, R., \& Rejowski, M. (2011). Abordaje teórico conceptual de la hospitalidad y sus contribuciones a la educación superior en turismo. Estudios y perspectivas en turismo, 20(6), 1464-1482.

López-Bonilla, J. M., \& López-Bonilla, L. M. (2004). Evolución y perspectivas del enfoque interdisciplinario en el estudio del turismo. Estudios turísticos, 160, 31-44. Obtenido de https://idus.us.es/xmlui/handle/11441/51747

López, M. C. T., \& Chávez, R. A. M. (2017). Evaluación del currículum real de la carrera Administración Turística y Hotelera. Revista Torreón Universitario, (14), 61-74.

Martínez, L. (2008). El currículo por competencias: un tema a debate. Pedagogía Universitaria. Editorial Universitaria, 13 (3), 1-17. Obtenido de http://cvi.mes.edu.cu/peduniv/index.php/peduniv/article/view/452/0

Martínez, R., Ruiz, R., \& Valladares, L. (2012). Innovación en la educación superior: hacia las sociedades del conocimiento. México D.F.: Fondo de Cultura Económica.

Maura, V. G. (2002). ¿Qué significa ser un profesional competente? Reflexiones desde una perspectiva psicológica. Revista Cubana de Educación Superior, 22(1), 45-53. 
Maura, V. G., \& Tirados, R. (2008). Competencias genéricas y formación profesional: un análisis desde la docencia universitaria. Revista iberoamericana de educación, 47, 185-209.

MEN. (2010). Niveles de la Educación Superior. Obtenido de: https://www.mineducacion.gov.co/1759/w3-article-231238.html

MEN. (2019). Sistema Nacional de Información de la Educación Superior (SNIES). Obtenido de https://www.mineducacion.gov.co/sistemasinfo/InformacionInstitucional/211868:Que-es-el-SNIES

Méndez-Giménez, A., Sierra-Arizmendiarrieta, B., \& Mañana-Rodríguez, J. (2013). Percepciones y creencias de los docentes de Primaria del Principado de Asturias sobre las competencias básicas. Revista de Educación 362, 737-761

Mochón, F. (2008). Economía y turismo (2a. ed.). Buenos Aires: Mc Graw Hill.

Mora, J.A. (2018). "Oportunidad del turismo comunitario en el desarrollo rural del posconflicto colombiano". Gastronomía, Hotelería y Turismo: Sostenibilidad e Inclusión (pp.23-38). Bogotá: Fundación San Mateo.

Mora Forero, J. A., \& Bohorquez Patiño, L. M. (2018). El turismo rural comunitario ¿Una oportunidad en el posconflicto colombiano?. Revista Internacional de Turismo, $\begin{array}{llllll}\text { Empresa } & y & \text { Territorio, } 2 \text { (2), 49-59. Obtenido de }\end{array}$ https://helvia.uco.es/handle/10396/17812

Mora Forero, J.A. (2019). El desarrollo rural colombiano, una mirada desde el turismo comunitario. Turpade, (10), 41-54. Obtenido de http://www.turpade.com/noticia.asp?IDArticulo=78\&IDGrupo=3

Mora, J. A., Yamova, O., \& Murtuzalieva, T. (2019). Community-Based Tourism as the Leading Approach to the Rural Development. In Sustainable Leadership for Entrepreneurs and Academics (pp. 503-510). Springer, Cham.

Nechar, M. C. (2007). La investigación y epistemología del turismo: aportes y retos. Revista Hospitalidade, 4(2), 79-95. Obtenido de https://www.revhosp.org/hospitalidade/article/view/234

Nechar, M. C., \& Netto, A. P. (2011). Implicaciones epistemológicas en la investigación turística. Estudios y perspectivas en turismo, 20 (2), 384-403. Obtenido de https://www.redalyc.org/pdf/1807/180717607007.pdf.

Panosso, A. (2007). Filosofía del turismo. Una propuesta epistemológica. Estudios y perspectivas en turismo,16(4), 389-402. Obtenido de https://www.redalyc.org/pdf/1807/Resumenes/Abstract_180713898001_2.pdf.

Pérez, Z. P. (2011). Los diseños de método mixto en la investigación en educación: Una experiencia concreta. Revista electrónica educare, 15(1), 15-29. Obtenido de https://www.redalyc.org/articulo.oa?id=1941/194118804003

Pimentel, T. D., de Carvalho, F. C. C., \& de Oliveira, M. C. B. (2017). Distribución de la oferta educativa (OET) y de las estructuras formales de investigación en turismo 
(EFIT) en Ecuador. Turismo y Sociedad, (21), 193-215. https://doi.org/10.18601/01207555.n21.09

Rivera Mateos, M.; Osuna Rodríguez, M. y Rodríguez García, L. (2017). Educación intercultural y culturas indígenas en América Latina: la licenciatura en Pedagogía de la Madre Tierra. Revista Iberoamericana de Educación Superior, 23, 163-182.

Rodrigues Leal, S. (2006). Madurez de la investigación científica en turismo, en Brasil y en el mundo. Estudios y perspectivas en turismo, 15(1), 81-91.

Salcedo, A. L. T., \& Suárez, L. P. (2005). Investigación en turismo y educación en la Universidad Autónoma del Estado de México. Teoría y Praxis, (1), 87-95. Obtenido de https://www.redalyc.org/pdf/4561/456145114007.pdf.

Santana, M. Á. (2005). Turismo, empleo y desarrollo. Revista de sociologia, 79-104. Obtenido de https://ddd.uab.cat/record/4845.

Torres Zamudio, M. (2015). Formation references in higher education. Area of knowledge: Tourism. Sophia, 11(2), 185-205. Obtenido de http://www.scielo.org.co/scielo.php?script=sci_arttext\&pid=S1794$89322015000200007 \& \operatorname{lng}=$ en\&tlng=es.

Universidad Externado de Colombia. (Noviembre de 2014). Proyecto Académico. Facultad de Administración de Empresas Turísticas y Hoteleras. Obtenido de https://www.uexternado.edu.co/wpcontent/uploads/2017/01/Proyecto_Academico_2014-FHot.pd

Wallingre, N. (2011). Avances en la construcción del conocimiento del turismo: Pensando la disciplina del turismo desde una perspectiva integral. Estudios y perspectivas en turismo, 20(1), 149-170.WTTC (2016). Travel \& Tourism economic impact 2016 world. Obtenido de https://www.wttc.org/media/files/reports/economic\%20impact\%20research/regions \%202016/world2016.pdf

Yániz, C. (2008). Las competencias en currículo universitario: implicaciones para diseñar el aprendizaje y para la formación del profesorado. Revista de docencia universitaria. (6), 1, 1-14. Obtenido de https://revistas.um.es/redu/article/view/10621. 